IRSTI 29.27.19

\title{
The plasma parameters and neutron yield at device of "Plasma focus"
}

\author{
A.M. Zhukeshov ${ }^{1, *}$, Zh.M. Moldabekov ${ }^{1}$ and A.Ioffe ${ }^{2}$ \\ Ial-Farabi Kazakh National University, 71, al-Farabi Ave, 050040, Almaty, Kazakhstan \\ ${ }^{2}$ Centre for Neutron Science at Heinz Maier-Leibnitz Zentrum, \\ Lichtenberg str.1, 85747, Julich, Germany \\ *e-mail: azhukeshov@gmail.com
}

\begin{abstract}
Present work presents calculations and experimental studies on the formation and dynamics of plasma on the "plasma focus" set up. Physical conditions were determined and critical parameters were calculated for estimating the neutron yield for kilojoule and megajoule set ups. The results of plasma diagnostics and flow formation in the CPA-30 and PF-4 set ups are also shown. A comparison of calculated data and experimental values is performed. The value of the neutron yield parameter is justified and the possible cause of the appearance of saturation is indicated. The rationale for the development of further research in the direction of creating conditions for thermonuclear fusion in installations of the plasma focus type is given. The neutron flux was observed on high-voltage gas discharges with a specific geometry of the electrodes. The result of measuring the anisotropy of the neutron yield along the axial $\mathrm{z}$ and radial $\varphi$ directions was equal to $\mathrm{Y}_{\mathrm{n}}(\mathrm{z}) / \mathrm{Y}_{\mathrm{n}}(\varphi) \sim 2 \div 3$, which contradicts the thermonuclear mechanism.
\end{abstract}

Key words: neutron yield, plasma focus, maximum discharge current, electrode, silver and indium foils.

PACS number: $29.40 .-n$.

\section{Introduction}

The tasks of creating powerful sources of neutron fluxes and X-ray radiation are relevant for a number of industries of production and medicine, but the problem of solving the problem of thermonuclear fusion is even more acute [4-5]. One of the new developing approaches in the creation of thermonuclear installations is the method based on plasma generation in installations of the plasma focus type. Plasma focus (PF) is a pulsed unsteady clotof hightemperature dense hot plasma. When using deuterium as working gas, the $\mathrm{PF}$ is a localized source of neutrons and hard radiation. However, when using other materials, such as boron, the synthesis reaction may be without a neutron yield, but in this case, the energy yield of the reaction will be different. Therefore, the study of the possibilities of using plasma focus in thermonuclear energy is very important.

The phenomenon of "plasma focus" was discovered in the middle of the twentieth century, independently to each other by N.V. Filippov
(USSR) [1] and J. Mather (USA) [2] in studies conducted under the program of controlled thermonuclear fusion. PF attracted interest by researchers, when the working chamber of the PF was filled with a rarefied isotope of hydrogen with deuterium, a powerful short impulse of fast neutrons and X-rays is generated inside the chamber of the discharge current. The discharge current usually measured in hundreds of kilo Amperes [3,4]. The first PF installations had an energy reserve of $50 \mathrm{~kJ}$. In this case, the neutron yield achieved at these facilities was $\sim 10^{9}$ neutrons per pulse. The neutron pulse duration of the PF is $t \sim 100 \mathrm{~ns}$. From the practical point of view, installations with PF are used as sources of neutrons and hard radiation for solving a number of scientific and technical problems: materials science and blanket tests for controlled thermonuclear fusion; neutron therapy; pumping laser media; interactions of powerful beams with plasma, etc. In recent years, the direction of creating devices that are more compact has been developing $[5,6]$. 
There is more than a dozen PF set up in the world: the PF-1000 set up (Warsaw, Poland) has a capacity of $1 \mathrm{MJ}$, the Tulip plasma set up with power from $4 \mathrm{~kJ}$ to $0.4 \mathrm{MJ}$ (Moscow, Russia), and others [7-8]. Previously, the authors conducted experiments on a pulsed plasma accelerator PF-30. The power of the CPA-30 set up is $35 \mathrm{~kJ}$, the discharge current is $450 \mathrm{kA}$, and the duration is 7 $\mu$ s. On this set up we have formed dense, discharge plasma clots, and studied their dynamics, as well as the basic laws of plasma beam focusing [9]. In particular, it has been shown that the dynamics of light and dense plasma flows differ significantly not only in flow rate but also in the role of ions in plasma acceleration and the whole structure of the plasma flow [10].

The use of focus plasma in thermonuclear reactors was considered in [11-12]. With an adequate level of understanding of these processes, new perspectives are emerging for the creation of a fusion reactor based on new data. Therefore, it is necessary both to study the possibility of creating an alternative type of thermonuclear reactor at the plasma focus installations and to conduct experiments on existing installations. In this paper, the problem is posed to theoretically calculate the parameters of the neutron yield and then compare it with the experiment.

\section{Theoretical part.}

A feature of the plasma focus-type installations is the dependence of the neutron yield on the energy E stored in a capacitor battery, and accordingly on the magnitude of the discharge current at the moment of pinching $\mathrm{I}_{\mathrm{p}}$ :

$$
\begin{gathered}
Y_{n}=10 E^{2} \\
Y_{n}=10^{-13} I_{p}^{4}
\end{gathered}
$$

The PF set up of the kilojoule range, the inductance of the discharge chamber and the plasma column can be neglected, and then the discharge inductance will be determined by the inductance of the battery and lead wires. The value of the maximum discharge current for these installations is found by the formula (2), taking into account the energy stored in the capacitor battery is equal to:

$$
I_{m}=\sqrt{\frac{2 E}{L}}
$$

Expressing the energy from equation (6), and substituting the resulting expression into equation (3), we obtain

$$
Y_{n}=2,5 \cdot I_{m}^{4} L
$$

From equation (6) it follows that the neutron yield for installations with kilojoule power is determined by the magnitude of the maximum discharge current.

The practical evaluation of formula (4), was calculated the neutron yield value for the PF-30 experimental setup. The capacitor battery consisted of 9 to 18 capacitors, each with a capacity of $3 \mu \mathrm{F}$ and an inductance of $10^{-7} \mathrm{H}$. The maximum battery voltage is $30 \mathrm{kV}$. The maximum neutron yield for an installation with a capacitor battery with a capacity of $27 \mu \mathrm{F}$ (9 capacitors) was $1.5 \cdot 10^{9}$ neutrons/pulse, and for a battery with a capacity of $54 \mu \mathrm{F}(18$ capacitors) consist $5.9 \cdot 10^{9}$ neutron/pulse.

The electron concentration can be found using the following expression for the electrodynamic model:

$$
n_{e}=\frac{I^{2} f_{0} \mu_{0} t}{4 \pi \varepsilon r^{2}}
$$

Where $I$ - current, $f_{0}$ - frequency, $\mu_{0}$ - magnetic constant, $r$ - the distance between the electrode, $\varepsilon-$ ionization energy. From equation (5) it follows that the concentration of electrons depends on the current and the distance between the electrodes, which is illustrated in Figure 1. One can see that the electron concentration decreases with increasing anode radius. The change in the current of the circuit also affects $n$, it is clear that an increase in the electron temperature is affected by an increase in the current, but at the same time, the pulse time decreases. If the maximum current value is $1 \mathrm{MA}$, the electron temperature will take its maximum value of $126 \mathrm{eV}$, and the pulse time will be a minimum of $1 \mu \mathrm{s}$. More accurate determination of the maximum electron temperature is necessary to calculate the optimum ratio of the anode and cathode [19]. The obtained data allows us to determine the values of the electron temperature when the anode radius varies from $0.25 \mathrm{~cm}$ to $2.25 \mathrm{~cm}$. 


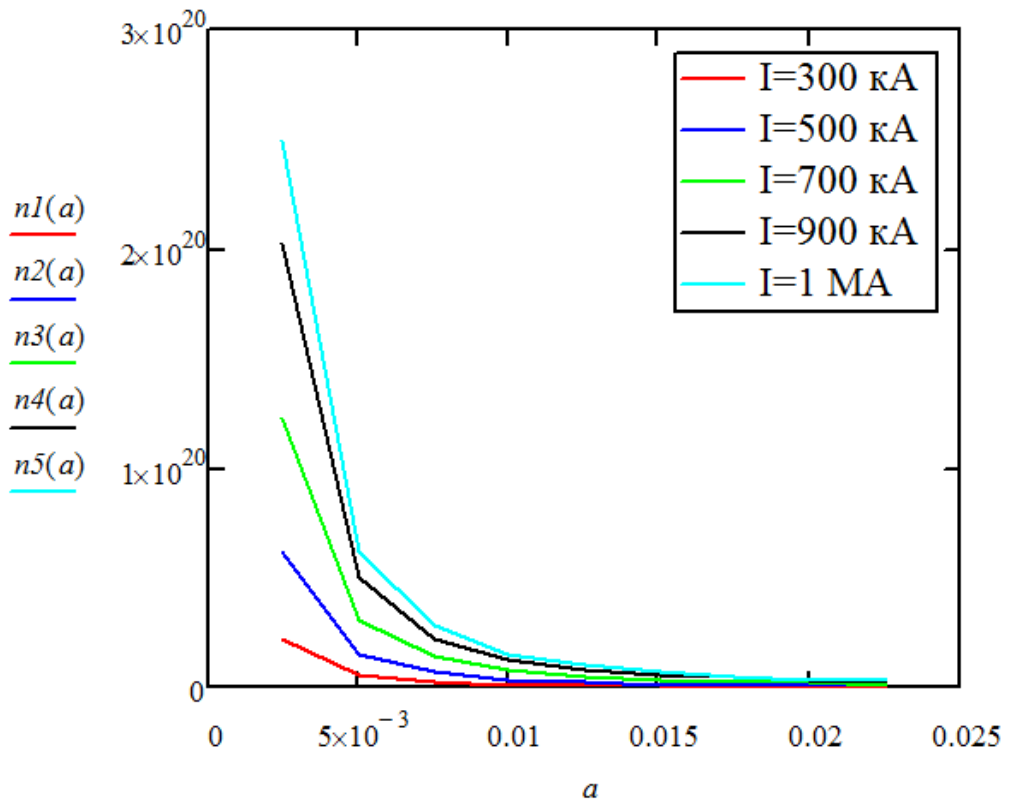

Figure 1- Changes in the concentration of electrons from the distance between the electrodes for different values of current

Thus, the maximum temperature and electron concentrations were $2.5 \cdot 10^{16} \mathrm{~cm}^{-3}$ and $126 \mathrm{eV}$, and the neutron yield for the PF-30 installation may be $5.9 \cdot 10^{9}$ neutron/pulse. Calculations on the electrodynamic model show that the electron concentration decreases with increasing anode radius.At the same time, an increase in current leads to an increase in concentration. In addition, it was found that an increase in the electron temperature is influenced by an increase in the current, but at the same time, the pulse time is reduced.

\section{Measurement of neutron yield}

To register a short duration neutron emission from Plasma Focus type pulsed sources at the P.N. Lebedev Physical Institute of Russian Academy of Sciences, a special detection system was developed, which is described in detail in [1516]. The task was to develop equipment for the registration of neutron radiation of the DD reaction for PF type set ups of relatively low intensity $\left(5 \cdot 10^{6}-2 \cdot 10^{7}\right.$ neutrons per discharge) under conditions of powerful fluxes of electromagnetic plasma radiation in the presence of intense electrical and magnetic interference, the calibration of this system. Measurement of neutron fluxes in these conditions can be successfully carried out using activation detectors. Activation detectors allow it possible to make measurements with a shift in time with respect to the moment of plasma generation after extinguishing of electromagnetic radiation and the cessation of interference [16-19]. Measurements produce on standard equipment and with greater accuracy than the accuracy achieved indirect measurements of the amplitude of the neutron pulse.

The halogen Geiger counters CTC-5 (CTC-6) used as sensors in these detectors (Figure 2) were wrapped with indium (or silver) foil and placed in the center of the cylindrical moderator box. The type of sensor used has a relatively large "dead time" $(\sim 100 \mu \mathrm{s})$. Therefore, when measuring large neutron yield, it is also important not to have an excessively large number of samples on the recording equipment in order to minimize the errors of the recording system due to the relatively large resolution time of the system on the counting input.

The activation detector variant with the CTC-5 sensors are characterized by lower sensitivity as compared to activation detectors of similar types, as the detector has a relatively small container moderator and the CTC-5 sensor itself has small dimensions compared, for example, the CTC-6 sensor with similar propertieswith, respectively, fewer counting on the measuring equipment. Measurements of neutron fluxes under these conditions can be successfully carried out using activation detectors $[7,14,15,16]$. 

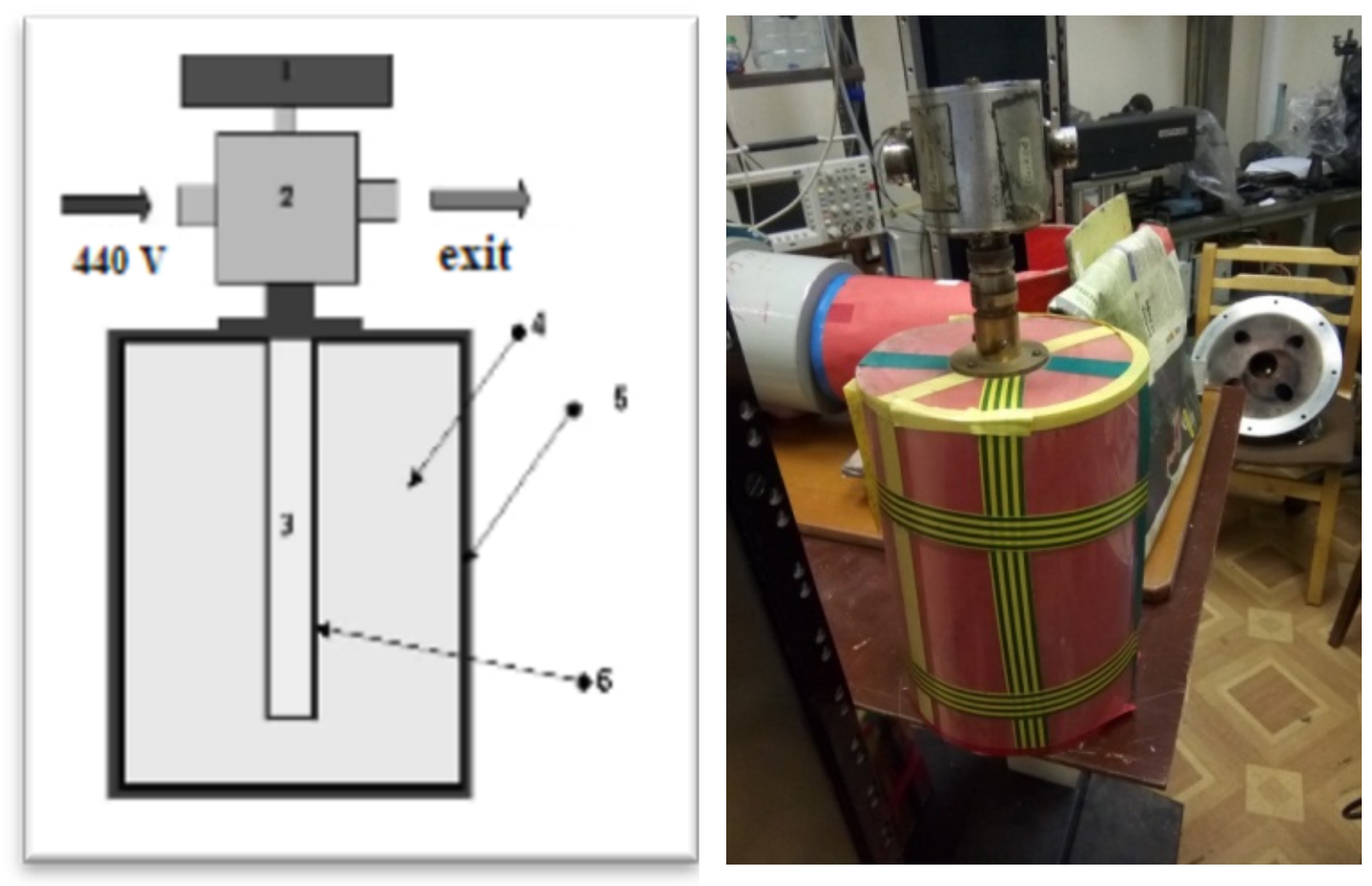

1 -battery power supply of the electric circuit; 2 - electrical diagram of the activation counter; 3 - halogen Geiger counter CTC-5 (CTC-6); 4 - cylindrical block of activation counter retarder; 5 - the layer of cadmium; 6 - activated foil (InorAg).

Figure 2 - Neutron detector developed in P.N. Lebedev Physical Institute of Russian Academy of Sciences.

Let the detector be irradiated with fast $(2.5$ $\mathrm{MeV}$ ) neutrons from a constant source of intensity I (neuter./sec.) located at the point from which the detector is visible at a solid angle $\Omega$. Thermal neutrons (after the process of slowing down fast neutrons) inside the moderator block activate foils that wrap the sensor. In general, the absolute neutrons yield:

$$
N+\Delta N-\bar{N}_{p h}^{\Delta t}=I\left(\frac{\Omega}{4 \pi}\right)\left[\varepsilon^{\prime} T^{\prime}\left(1-e^{-t_{1} / T^{\prime}}\right) e^{-t_{2} / T^{\prime}}\left(1-e^{-\Delta t / T^{\prime}}\right)+\varepsilon^{\prime \prime} T^{\prime \prime}\left(1-e^{-t_{1} / T^{\prime \prime}}\right) e^{\left.-t_{2} / T^{\prime \prime}\left(1-e^{-\Delta t / T^{\prime \prime}}\right)\right]}\right.
$$

The indices "one stroke" and "two strokes" refer respectively to the parameters for indium isomers $\mathrm{In}^{116}$ and $\mathrm{In}^{116 \mathrm{~m}}$ or the isotopes $\mathrm{Ag}^{108}$ and $\mathrm{Ag}^{110}$. Description of other parameters in the formula (6) is given in $[8,9]$.
The results of experiments on measuring the neutron yield at the PF-4 installation [7] are shown in Figures 3 and 4 for indium and silver foil respectively, used as a fast neutron moderator. 


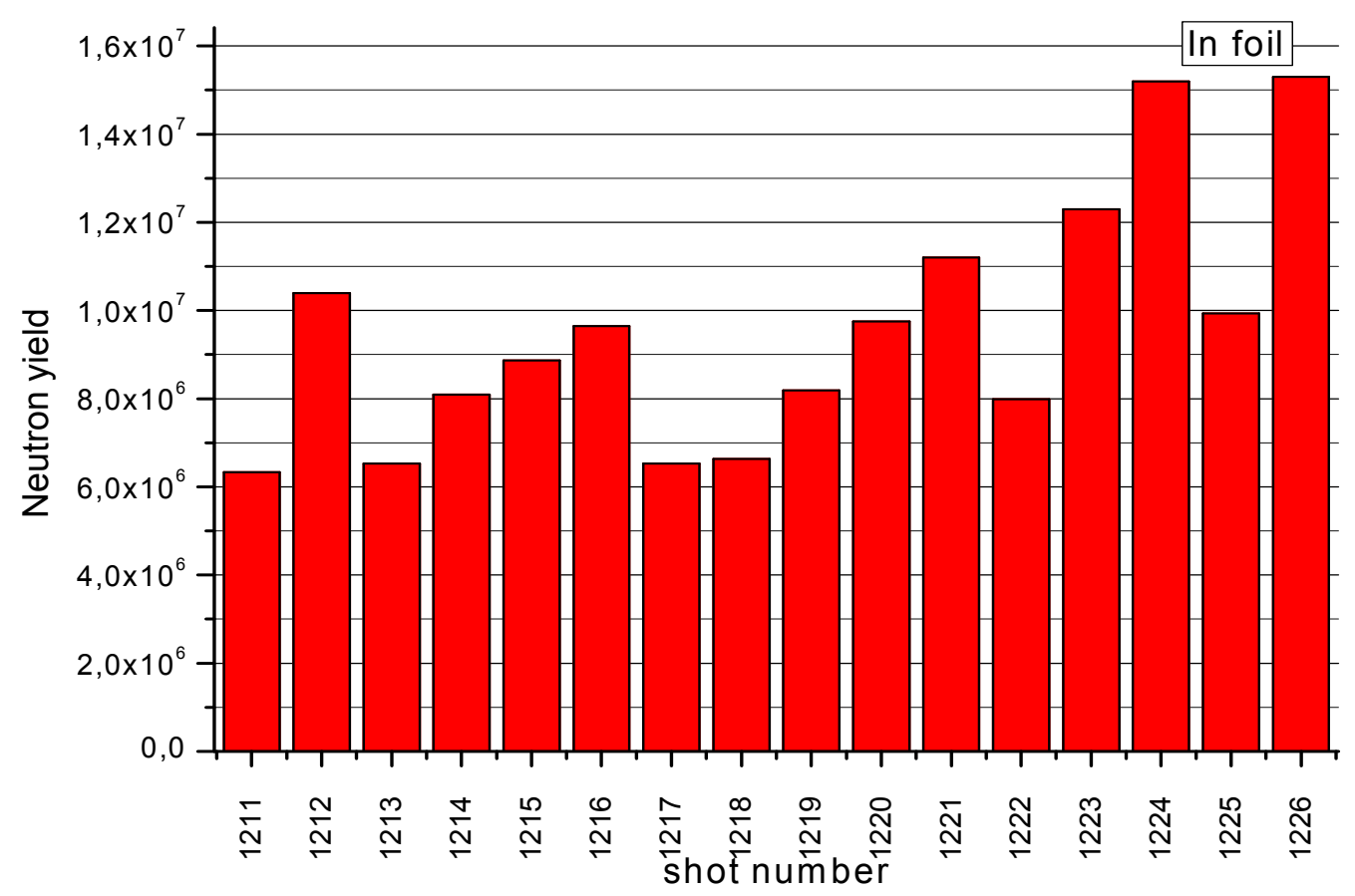

Figure 3 - PF-4 neutron yield with indium foil

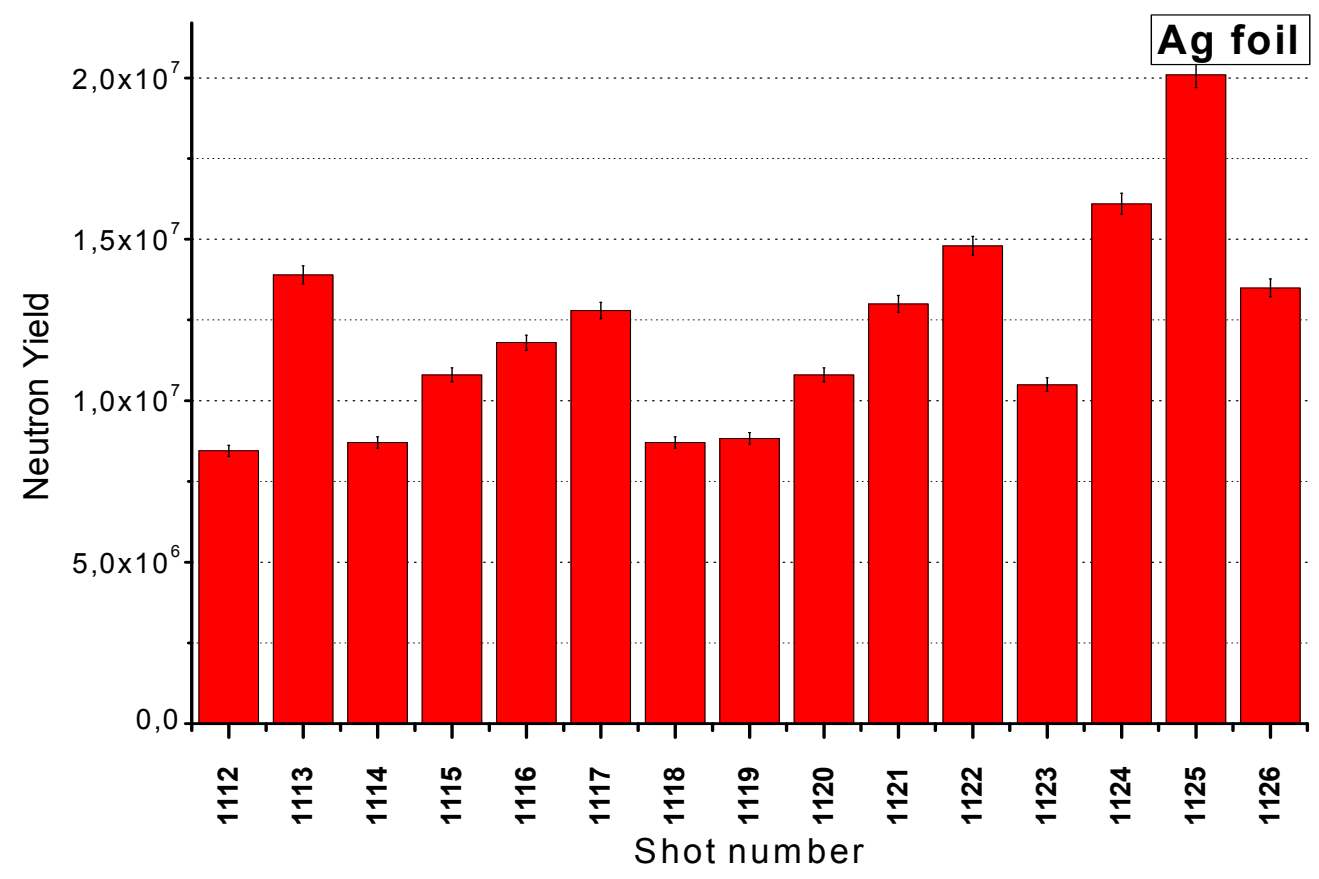

Figure 4 - PF-4 neutron yield with silver foil. 


\section{Conclusions}

The results of calculations and experiments shown that for installations like plasma focus PF-30 and PF-4 it is possible to obtain discharge currents of hundreds of kiloamperes and current rise rates up to $\sim 10^{11} \mathrm{~A} / \mathrm{s}$. The current data determined from the experiments and the plasma parameters agree with the calculated values given above. At the same time, the neutron yield was calculated on the basis of the thermodynamic model, i.e. a priori assuming its thermonuclear origin. But a review of the experimental facts suggests that neutron emission may be associated with other conditions and is not characteristic only of "plasma focus". For example, the neutron flux was also observed on high-voltage gas discharges with a specific geometry of the electrodes, creating field strength sufficient for "runaway of electrons" [13]. In this case, the typical times of processes are counted in hundreds of nanoseconds, which are also observed for a plasma focus.
Previously reported experimental work that when using deuterium gas in the PF, neutron yield is observed with the energy distribution along the axis of the system and energy of $2.45 \mathrm{MeV}$.Neutron radiation had an isotropic distribution and its duration was of the order of tens of nanoseconds.It was assumed that these neutrons are of thermonuclear origin, which leads to isotropic neutron radiation. However, the result of measuring the anisotropy of the neutron yield along the axial $\mathrm{z}$ and radial $\varphi$ directions was equal to $\mathrm{Y}_{\mathrm{n}}(\mathrm{z}) / \mathrm{Y}_{\mathrm{n}}(\varphi) \sim 2 \div 3$, which contradicts the thermonuclear mechanism. In addition, the average energy of neutrons in the axial direction was greater than the energy of thermonuclear neutrons $(2.45$ $\mathrm{MeV}$ ). This fact indicates that it is worth considering the non-thermal mechanism of neutron emission.

Thus, today, both thermonuclear and beam emission options are possible, but the details and mechanism of ion acceleration still need to be clarified.

\section{References}

1. V. I. Krauz. Progress in plasma focus research and applications // Plasma Physics and Controlled Fusion. 2006. -Vol. 48, No.12. - P. B221.

2. J. W. Mather. Formation of the high-density deuterium plasma focus // Phys. Fluids. - 1965. - Vol. 8. - P. 366.

3. N.V. Filippov, T.I. Filippova, I.V. Khutoretskaia, V.V. Mialton, V.P. Vinogradov. Megajoule scale plasma focus as efficient X-ray source // Physics Letters A. - 1996. - Vol. 211, No. 3. - P. 168-171.

4. Y.C. Francis, R. Eskridge, J. Smith, A. Martin, J. Cassibry, S.T. Wu. A plasma accelerator concept for application to magnetized target fusion // Z-Pinches Conference. - Florence: Alabama university press. - 2006. - P. 456 $-467$.

5. L. Soto, C. Pavez, J. Moreno, M. Barbaglia, A. Clausse. Nanofocus: an ultra-miniature dense pinch plasma focus device with submillimetric anode operating at 0.1 J // Plasma Sources Sci. Technol. - 2009. -Vol.18. - P. 015007.

6. A. Skoulakis, G. C. Androulakis, E. L. Clark1 et al. Portable pulsed neutron generator // International Journal of Modern Physics: Conference Series. - 2014. - Vol. 27. - P. 1460127.

7. V.Ya. Nikulin, S.N. Polukhin, A.A. Tikhomirov. A Simple criterion for the snowplowing efficiency of the working gas in a kJ plasma focus // Plasma Physics Reports. - 2005. - Vol. 31, No. 7. - P.591-595.

8. A.M. Zhukeshov, B.M. Ibraev, Sh.G. Giniyatova, B.M. Useinov, V.Ya. Nikulin, A.T. Gabdullina, A.U. Amrenova. Parameters calculation and design of vacuum camera for «Plasma Focus» facility // International Journal of Mathematics and Physics. - 2016. - Vol.7, №1. - P. 137-140.

9. A. Zhukeshov, A. Amrenova , A.Gabdullina, Z. Moldabekov. Calculation and analysis of electrophysical processes in a high-power plasma accelerator with an intrinsic magnetic field // Technical physics. - 2019. - Vol. 62, No.3. - P. $342-347$

10. L. Soto. New trends and future perspectives on plasma focus research // Plasma Phys. Control. Fusion. - 2005. - Vol. 47. - P. A361-A381.

11. F. Castillo, M. Milanese, R. Moroso, J. Pouzo. Evidence of thermal and non-thermal mechanisms coexisting in dense plasma focus D-D nuclear reactions // J. Phys. D: Appl. Phys. - 2000. - Vol. 33, No. 2. - P.1.

12. M.I. Lomaev, B.A.Hechaev, B.N. Padalko. Neutron emission at nanosecond discharge in deuterium in an inhomogeneous electric field // JTPh. - 2012. - Vol. 82, No.1. - P. 126-133 (in Russian).

13. O. N. Krokhin, V.Ya. Nikulin, M. Scholz, I.V. Volobuev. The measurements of neutron emission on plasma focus installations with energy ranging from 4 to $1000 \mathrm{~kJ} / /$ Proc. of 20th Symp. on Plasma Physics and Technology. Prague. - 2002. - P. 61 
14. M. Scholz, B. Bieńkowska, M. Borowiecki, I. Ivanova-Stanik, L. Karpiński, W. Stępniewski, M. Paduch, K. Tomaszewski, M. Sadowski, A. Szydłowski, P. Kubeš, J. Kravárik. Status of a mega-joule scale Plasma-Focus experiments // Nukleonika. - 2006. - Vol.51, No.1. - P. 79-84.

15. O.N. Krokhin, V.Ya. Nikulin, I.V. Volobuev. Compact activation detectors for measuring of absolute neutron yield generated by powerful pulsed plasma installations // 21nd Symposium on Plasma Phys. And Techn., June 2004, (Praha, Czech. Rep.). Czech. J. Phys. - 2004. - Vol. 54. - P.1.

16. N. A. Vlasov. Neutrons. -1955. Moscow: State ed. technical - theoretical literature (in Russian)

17. A.M. Zhukeshov, B.M. Ibraev, B.M. Useinov, Sh.G. Giniatova. Pulsed plasma flow interaction with a steel surface //High Temperature materials and processes. - 2015. - Vol. 19, No.2. - P. 113-119.

18. H. Rahal, C. Deutch, M.M. Gombert. Temperature dependent quantum pair potentials and ionization in Helium like plasma //Physical Sciences and Thechnology. - 2017. - Vol.4, No. 2 - P. 29-46.

19. E. Ishitsuka, E. Kenzhina. Evaluation of tritium release curve in primary coolant of research reactor // Physical Sciences and Thechnology. - 2017. - Vol.4, No. 1. - P. 27-33.

20. B.M. Ibraev, A.M. Zhukeshov, A.T. Gabdullina, A.U. Amrenova. Research of plasma accelerator KPU-30 // Int. J. of Math. and Physics. - 2012. - Vol. 3, No.1. - P. $50-53$ 